\title{
Uma Abordagem para a Recomendação Inteligente de Conteúdo Web na Criação de Materiais e Objetos de Aprendizagem
}

\author{
Clarivando F. Belizário Júnior, Fabiano A. Dorça
}

Faculdade de Computação - Universidade Federal de Uberlândia (UFU)

Uberlândia - MG - Brasil

clarivando@ufu.br, fabianodoreufu.br

\begin{abstract}
Resumo. A criação de cursos online em larga escala adaptáveis aos estilos cognitivos e de aprendizagem dos estudantes é um problema desafiante e pendente. Muitas abordagens tratam apenas da adaptação desses cursos, e as poucas que tratam da criação, exigem do usuário conhecimento técnico e de domínio do conhecimento na construção de objetos de aprendizagem. Neste artigo, uma nova abordagem é modelada para lidar com esses desafios. Nela, dado um programa de curso, suas seções são mapeadas em conceitos representados em ontologias descritas na linguagem OWL da Web Semântica. Esses conceitos são utilizados na busca inteligente por objetos de aprendizagem e por recursos web anotados em $R D F$, que podem ser utilizados na criação de objetos de aprendizagem.
\end{abstract}

Abstract. The creation of online adaptive courses, which consider cognitive and learning styles information about students, is a challenging problem. Many approaches consider only the adaptation of these courses, and the few that deal with the creation of them require tutor expertise and domain knowledge in the construction of learning objects. In this article, a new approach is modeled to deal with these challenges. In the proposed, given a course design, its sections are mapped to concepts represented in ontologies described in OWL language of the Semantic Web. These concepts are used in an intelligent search for web resources with RDF metadata which comply with the lesson domain, automating the authoring process of new learning objects.

Palavras-chave: Web Semântica, Objetos de Aprendizagem, Busca Semântica

\section{Introdução}

Um dos maiores desafios da Educação é adaptar o processo de ensino-aprendizagem aos estilos cognitivo e de aprendizagem de cada aluno, pois no contexto da sala de aula, as estratégias de ensino são voltadas para a turma. Há poucos anos, a informática tornou-se uma grande aliada da Educação na solução desse desafio com a utilização de hipermídias adaptativas capazes de adaptar conteúdo educacional digital às preferências de aprendizagem de cada estudante. Essas hipermídias podem ser integradas a Sistemas de Gerenciamento de Aprendizagem, como o Moodle, permitindo a criação de cursos online.

Se as máquinas compreendessem a linguagem humana, esses cursos seriam criados de maneira totalmente automatizada com base num simples programa de curso. 
Por outro lado, a criação desses cursos pelo usuário tende a ser um processo demorado que coloca o esforço no conhecimento técnico em vez do domínio de instrução. Além disso, a busca por recursos web para compor recursos educacionais poucas vezes retorna os melhores conteúdos. Esses são alguns dos desafios da computação que precisam ser solucionados para a efetivação do uso de tecnologias na Educação.

Para enfrentar esses desafios, propõe-se uma abordagem para modelar ferramentas de criação de cursos online usando a Web Semântica, exigindo, assim, o menor esforço possível do criador do curso. Embora a Web possa ter informações suficientes para compor um curso online, elas não são compreendidas pelas máquinas. A Web Semântica surgiu como uma extensão da Web tradicional para superar essa limitação permitindo que os computadores pudessem compreender as informações publicadas na internet.

No contexto deste trabalho, considera-se que um programa de curso é dividido em capítulos que por sua vez são divididos em seções. O material de aprendizagem é formado por um conjunto de OAs de tipos diferentes, por exemplo, exercício, questionário e conclusão. A ferramenta que implementar esta abordagem deve criar um material de aprendizagem para cada seção do programa de curso. Para isso, o primeiro passo é mapear cada seção do programa de curso para um conceito de alto nível, que pode ser decomposto em outros conceitos de nível inferior, representado na ontologia do domínio de conhecimento do curso. Esses conceitos de alto nível representam as metas de aprendizagem dos aprendizes no início do curso e devem ser utilizados na busca pelos recursos web que comporão o curso online. Selecionaram-se quatro trabalhos da literatura que utilizam abordagens diferentes para recomendar (buscar por) OAs usando ontologias. Essas abordagens, que consideram as competências e os estilos cognitivos dos aprendizes, serão implementadas e testadas para ser selecionada a melhor no contexto da abordagem proposta neste trabalho.

Como nem sempre o usuário encontra materiais e objetos de aprendizagem da Web prontos para compor seu curso, ele deve criá-los do zero. A abordagem proposta neste trabalho modela a criação de OAs para auxiliar o usuário na realização dessa tarefa. Para isso, há alguns métodos de buscas na Web Semântica, mas não há nenhum estudo comparativo da eficiência deles no contexto da criação de recursos educacionais. Portanto, comparar-se-ão esses métodos para escolher o mais eficiente no contexto educacional e, em seguida, utilizá-lo na criação de um algoritmo de busca semântica a ser utilizado na abordagem proposta. $\mathrm{O}$ algoritmo deve executar a busca em conteúdos web anotados na linguagem RDF (Resource Description Framework). O resultado desse algoritmo é um conjunto de recursos web apropriados para compor cada objeto de aprendizagem a ser criado pelo usuário. Há trabalhos recentes que permitem a geração semiautomática de metadados de OAs derivados de capturas multimídia (ARAÚJO et al., 2014) e conteúdos da Wiki (MENOLLI; MALUCELLI; REINEHR, 2011), mas não derivados de conteúdos web anotados em RDF. Portanto, na abordagem apresentada neste trabalho investigam-se maneiras de automatizar a criação de metadados de OAs no padrão IEEE-LOM derivados de recursos web anotados em RDF.

É importante ressaltar que novos conteúdos são publicados todos os dias na rede pelo grande público e, portanto, acredita-se que vá haver sempre a necessidade de criação de novos OAs e, consequentemente, a criação e atualização de ontologias para representarem novos conteúdos, por isso, a criação de OAs é um processo contínuo que 
deve ser automatizado, dado que na maioria das vezes os objetos recomendados estarão ultrapassados.

Assim, na ferramenta web que implementar a abordagem proposta, o usuário entrará apenas com o programa de curso e informações de filtragem que julgar necessárias (como palavras-chave e os formatos dos OAs que comporão cada seção) e a ferramenta web usará ontologias para mapear cada seção do curso descrita em linguagem natural para conceitos de alto nível, os quais são utilizados na busca inteligente por OAs que poderão compor cada material de aprendizagem (seção) do curso. Caso o usuário julgue algum objeto inadequado, poderá criar seu próprio objeto de aprendizagem usando a recomendação inteligente de conteúdo realizada pelo algoritmo de busca semântica em arquivos RDF. Os OAs criados pelo usuário deverão ser adicionados a repositórios de OAs descritos no padrão IEEE-LOM para promover a reusabilidade.

Portanto, a abordagem proposta contribuirá para a criação de materiais e objetos de aprendizagem exigindo o menor esforço possível do usuário e permitirá a reusabilidade desses recursos educacionais por diversos profissionais da Educação. Ao suprir as expectativas desses profissionais, esta abordagem mostra-se uma solução promissora para a efetivação do uso de tecnologias na Educação.

\section{Referencial Teórico}

\subsection{A Web Semântica}

A Web Semântica (BERNERS-LEE et al., 2001) surgiu como uma extensão da Web tradicional para permitir a compreensão dos dados da internet por computadores. Diversos estudos estão sendo realizados para a consolidação das camadas RDF (Resource Description Framework), RDF-S e ontológica pertencentes à Web Semântica. A camada RDF pode ser considerada uma camada de base composta por triplas em XML (Extensible Markup Language) formadas por sujeito, predicado e objeto capazes de especificar a semântica de metadados facilitando a busca por recursos na Web.

A RDF-S (RDF-Schema) tem o objetivo de descrever propriedades e classes, bem como suas hierarquias. Essa camada é utilizada em conjunto com a RDF, visando ainda mais o enriquecimento semântico. A camada ontológica utiliza a linguagem OWL (Web Ontology Language) para instanciar ontologias na Web. Essa camada fornece vocabulário adicional com uma semântica formal para potencializar a interpretação por máquinas do conteúdo da Web.

De modo geral, essas camadas utilizam metadados (descritos em RDF ou OWL) para descrever os relacionamentos semânticos entre os conteúdos da Web. A linguagem SPARQL é a linguagem padrão da Web Semântica usada para efetuar consultas sobre gráficos RDF e OWL. Como essas consultas consideram a semântica dos conteúdos, elas são bem mais eficientes que as consultas realizadas na Web tradicional. 


\subsection{Padrões de metadados de objetos de aprendizagem}

Os diversos conteúdos da Web Semântica podem ser usados na criação de objetos de aprendizagem. O padrão IEEE-LOM (DUVAL, 2002) é reconhecido internacionalmente por facilitar a busca e criação de OAs. O padrão IEEE-LOM é parte integrante dos padrões OBAA (VICCARI et al., 2010) e SCORM (ADL, 2001). O padrão de metadados OBAA estende o padrão IEEE-LOM e fornece interoperabilidade entre plataformas de hardware para lidar com o contexto educacional brasileiro. O SCORM (Sharable Content Objects Reference Model) é um padrão de referência que assegura a interoperabilidade entre diferentes sistemas de gerenciamento da aprendizagem. Os metadados de OAs descritos nesses padrões são armazenados em repositórios de OAs, permitindo a reusabilidade desses recursos educacionais pelas ferramentas de recomendação de OAs.

\section{Trabalhos Relacionados}

O uso de ontologias parece promissor no ensino, e há trabalhos recentes que as utilizam para a recomendação de OAs. Di Martino (2009a) utilizou técnicas semânticas combinadas com técnicas de casamento de esquemas (schema matching) para buscar por OAs relevantes (no padrão SCORM) usando ontologias de um dado domínio.

Castro e Satler (2010) utilizaram técnicas de mineração de dados no repositório de OAs AGORA para gerar regras (conhecimento) na linguagem SWRL da Web Semântica. Essas regras representadas em uma ontologia permitem classificar os OAs em ativos, passivos e muito passivos. Os autores mostram que esses resultados são importantes para o sequenciamento de OAs, e essas técnicas de mineração de dados podem ser melhoradas para a busca e rotulação de OAs.

Jácome Júnior, Mendes Neto e Da Silva (2012) apresentam uma abordagem baseada em algoritmo genético (AG) para a recomendação de objetos de aprendizagem sensível ao contexto do estudante. Agentes de software são combinados com ontologias de descrição de perfis (estático e dinâmicos) dos alunos e com o AG para a recomendação de conteúdos educacionais de maneira autônoma.

Gaeta et al. (2013) utilizaram ontologias para modelar domínios do conhecimento considerando duas relações principais entre conceitos. Na primeira relação, um conceito ou um grupo de conceitos faz parte de um outro conceito de alto nível, assim chamado por conter um ou mais conceitos. Na segunda relação, um conceito é requerido por outro, isso implica que o conceito requerido deve ser aprendido antes desse outro. Os conceitos de alto nível que forem a meta final do processo de aprendizagem devem ser identificados manualmente pelo usuário na ontologia. Então, um algoritmo analisa essa ontologia e o modelo cognitivo do estudante para gerar um caminho de aprendizagem formado pelos conceitos necessários para atingir as metas de aprendizagem. Em seguida, outro algoritmo cria uma sequência de OAs associada a esses conceitos. Esse algoritmo utiliza um algoritmo guloso para minimizar o número de OAs dessa sequência, e recomenda os OAs que melhor satisfazem as preferências de aprendizagem do estudante.

$\mathrm{Na}$ Tabela 1, são apresentadas as principais técnicas de busca e recomendação de OAs no contexto dos quatro trabalhos apresentados anteriormente. Testar-se-ão essas técnicas para escolher a melhor no contexto da abordagem proposta neste trabalho. 
As técnicas baseada em schema matching e algoritmo genético foram testadas em metadados do padrão SCORM, enquanto que as técnicas baseadas em regras de inferência e no algoritmo guloso foram testadas em metadados do padrão IEEE-LOM.

Tabela 1: Técnicas de recomendação de OAs

\begin{tabular}{|c|c|c|}
\hline Literatura correlata & Técnicas de recomendação de OAs & $\begin{array}{c}\text { Padrões de } \\
\text { metadados }\end{array}$ \\
\hline Di Martino (2009b) & Schema matching & SCORM \\
\hline Castro e Satler (2010) & $\begin{array}{c}\text { Regras de inferência (criadas pela } \\
\text { Mineração de Dados) }\end{array}$ & IEEE-LOM \\
\hline $\begin{array}{c}\text { Jácome Júnior, Mendes } \\
\text { Neto e Da Silva (2012) }\end{array}$ & Algoritmo Genético & SCORM \\
\hline Gaeta et al. (2013) & Algoritmo Guloso & IEEE-LOM \\
\hline
\end{tabular}

Sabe-se que muitas vezes os usuários não encontram os OAs adequados para compor seus cursos online. Nessas situações, eles criam seus próprios OAs usando recursos da Web. Porém, poucas vezes a Web tradicional retorna os recursos adequados para compor um dado objeto de aprendizagem. Para solucionar esse problema, trabalhos recentes utilizam a Web Semântica para recomendar recursos web descritos em RDF de maneira mais eficiente. Os algoritmos de busca na Web Semântica são de propósito geral e, portanto, podem ser testados em diversos contextos. Contudo, a literatura carece de trabalhos que avaliem e comparem esses algoritmos no contexto do e-learning. Para proceder com essa tarefa, implementar-se-ão e avaliar-se-ão os principais algoritmos de busca na Web Semântica, quais sejam: OntoRank, TripleRank, RareRank e Re-Rank semântico.

Em seguida, escolher-se-á o melhor no contexto da nova abordagem apresentada. O OntoRank (FININ et al., 2005) é o algoritmo usado pelo Swoogle (DING et al., 2004) na busca por conteúdo web anotado em RDF. Esse algoritmo é fundamentado no modelo rational surfer. Um agente racional navega de um Documento da Web Semântica (SWD) para outro com uma probabilidade constante ou salta para um SWD aleatório. Esse agente utiliza ontologias para compreender os termos dos documentos web visitados.

O algoritmo TripleRank (FRANZ et al., 2009) captura a semântica latente de dados da Web Semântica, por meio de métodos estatísticos, a fim de produzir descrições mais detalhadas desses dados.

O RareRank (WEI; BARNAGHI; BARGIELA, 2011) é um agoritmo utilizado para ranquear entidades em sistemas de busca semântica. Esse algoritmo simula o comportamento racional de pesquisadores humanos na busca por conteúdo da literatura científica.

O algoritmo Re-Rank semântico (WANG et al., 2011) utiliza similaridade semântica para melhorar os resultados da busca. Os resultados candidatos retornados por uma ferramenta de busca são ranqueados novamente (re-rank) comparando-os com a consulta.

No contexto da nova abordagem proposta, dentre esses algoritmos, o escolhido será utilizado na busca por recursos web que poderão compor OAs. Esse processo envolve a criação de metadados no padrão IEEE-LOM usando os metadados em RDF 
dos recursos web. A literatura carece de trabalhos que tratam essa questão, que permanece em aberto. Resolver essa questão é importante porque a descrição de objetos em um padrão específico do contexto educacional permite a construção de ferramentas de busca direcionadas a esse contexto e, portanto, mais otimizadas. Portanto, investigarse-ão possíveis mapeamentos entre metadados RDF e metadados descritos no padrão IEEE-LOM.

\section{Abordagem Proposta}

Propõe-se uma abordagem para modelar ferramentas de criação de cursos online usando ontologias da Web Semântica. Essas ontologias lidam com a linguagem natural dos programas de curso para identificar neles os conceitos que representarão os objetivos de aprendizagem. Esses conceitos são usados na busca por OAs ou, caso os objetos não sejam encontrados, na busca por recursos web anotados em RDF, que podem ser utilizados na criação de OAs.

A arquitetura dessa abordagem é apresentada na Figura 1. As seções do curso representam conceitos relevantes de um domínio do conhecimento representado por uma ontologia, a qual é utilizada para gerar um material de aprendizagem (conjunto de OAs) para cada seção do programa de curso. Um programa de curso é dividido em capítulos, os quais são divididos em seções. Cada material de aprendizagem é formado por um conjunto de OAs de tipos diferentes. Esses tipos são os mesmos utilizados no mecanismo flexível de recomendação de OAs proposto por Graf, Kinshuk e Ives (2010). Isso significa que trabalhos no estado da arte baseados nesses tipos de OAs, como a abordagem proposta por Silva e Dorça (2014), podem ser facilmente combinados com a nova abordagem proposta neste trabalho para se criar um sistema tutorial inteligente completo, com autoria de conteúdo (semi)automatizada e personalização de cursos online com base nos estilos de aprendizagem dos estudantes.

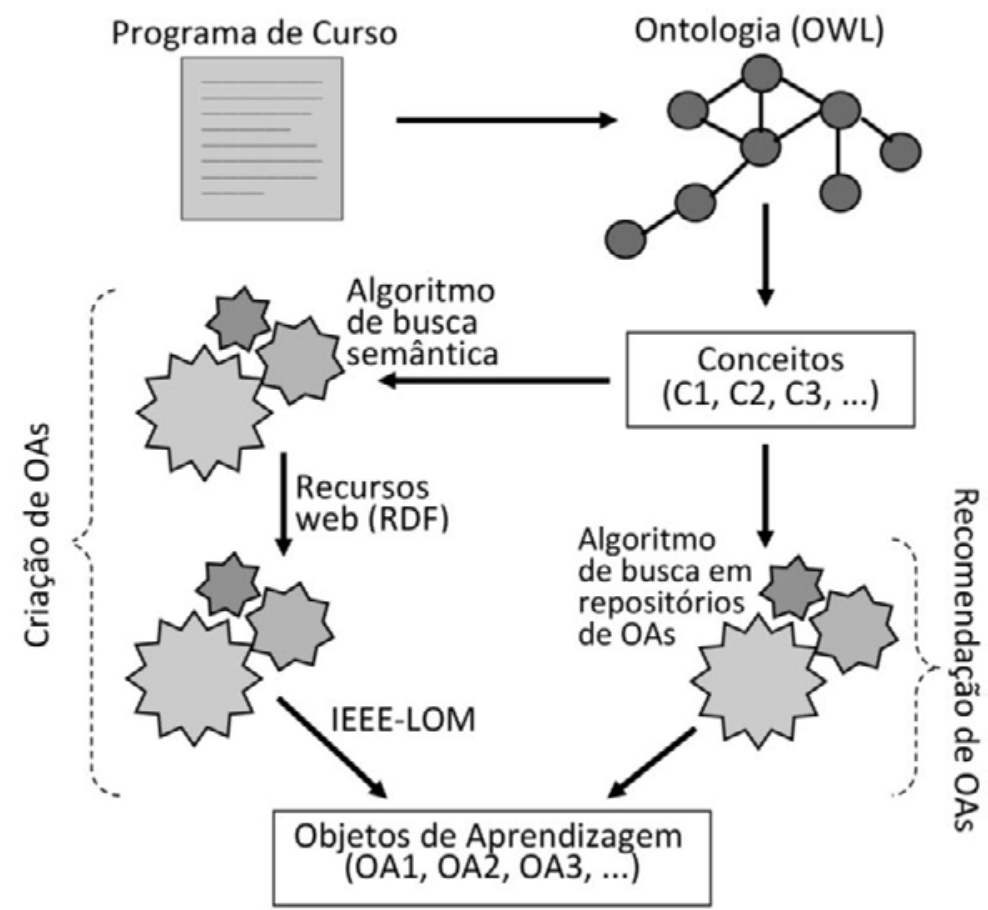

Figura 1: Modelo para a recomendação e criação de OAs 
Um diferencial da abordagem apresentada é lidar com a linguagem natural dos programas de curso, dos quais são derivados os conceitos a serem aprendidos pelo estudante. Para isso, o primeiro passo é mapear a linguagem natural para conceitos representados em uma ontologia anotada em OWL, que é uma linguagem da Web Semântica que as máquinas entendem. Embora cada conceito seja parecido com a linguagem natural da sua seção de curso correspondente, acredita-se que esse mapeamento não seja simples, pois os computadores não compreendem a linguagem natural, por isso, usar-se-ão ontologias associadas a um domínio do conhecimento, capazes de associar um conceito de alto nível para cada seção do programa de curso. Utilizar-se-á a linguagem SWRL (Semantic Web Rule Language) para implementar as regras que farão a inferência desses conceitos usando a ontologia. Para recomendar OAs usando esses conceitos, testar-se-ão as quatro técnicas identificadas nos trabalhos relacionados a este. Em seguida, escolher-se-á a técnica mais eficiente no contexto da abordagem proposta neste trabalho.

Di Martino (2009a) propôs uma ferramenta que aplica técnicas de schema matching entre um esquema de origem e um esquema alvo. O esquema de origem é uma ontologia que modela um domínio do conhecimento e agrega exatamente os conceitos a serem aprendidos. $\mathrm{O}$ esquema alvo é um arquivo xml que representa os conceitos extraídos de repositórios de OAs. Os detalhes da criação desse segundo esquema são apresentados em Di Martino (2009b). Para implementar essa recomendação de OAs no contexto da abordagem deste trabalho, utilizar-se-ão os conceitos gerados com base no programa de curso para representar o esquema de origem, mantendo entre esses conceitos os mesmos relacionamentos presentes na ontologia usada para gerá-los. $\mathrm{O}$ esquema alvo será o mesmo que foi utilizado pelo autor. Assim, as técnicas de schema matching usadas pelo autor poderão ser testadas no escopo da abordagem deste trabalho.

Para testar as técnicas de mineração de dados (em repositórios do padrão IEEELOM) propostas por Castro e Satler (2010), utilizar-se-ão os conceitos gerados com base no programa de curso mantendo os mesmos relacionamentos da ontologia de domínio da qual esses conceitos foram derivados, formando assim, uma ontologia composta apenas pelos conceitos a serem aprendidos pelo estudante. As regras de inferência geradas pelas técnicas de mineração de dados a serem testadas serão inseridas nessa ontologia. Assim, essas técnicas serão avaliadas na recomendação de OAs. Investigar-se-ão trabalhos no estado da arte para filtrar esses OAs com base nos estilos de aprendizagem e cognitivo dos estudantes.

Jácome Júnior, Mendes Neto e Da Silva (2012) utilizaram um algoritmo genético (AG) para a recomendação de OAs do padrão SCORM com base nas características desses OAs relacionadas com o perfil do estudante representado por uma ontologia. No contexto da nova abordagem proposta, essa ontologia também modelará os conceitos a serem aprendidos pelo estudante. Assim, o AG a ser implementado será avaliado na recomendação de OAs com base nos estilos de aprendizagem e cognitivo dos aprendizes.

A última técnica a ser testada envolve a aplicação de um algoritmo guloso (GAETA et al., 2013) em uma sequência de OAs relacionados aos conceitos a serem aprendidos pelos estudantes. Esse algoritmo minimiza o número de OAs dessa sequência para recomendar os OAs que satisfazem as preferências de aprendizagem dos 
estudantes. Assim como no trabalho de Gaeta et al. (2013), esses conceitos serão modelados por uma ontologia. Embora o algoritmo guloso não seja muito preciso, ele é rápido, por isso, é importante testá-lo no contexto da abordagem proposta.

Como nem sempre os OAs adequados são encontrados, a abordagem apresentada permite que o usuário crie seus próprios OAs usando os conteúdos da Web Semântica. Para isso, os mesmos conceitos utilizados na busca por OAs, são usados na busca por conteúdos web anotados em RDF, que servirão para compor os OAs. Testarse-ão quatro algoritmos de busca diferentes, o OntoRank, o TripleRank, o RareRank e o Re-Rank semântico, para escolher o melhor no contexto do ensino-aprendizagem.

O conteúdo web, propriamente dito, retornado por esses algoritmos pode ser mapeado diretamente para o conteúdo de OAs, mas o mapeamento de metadados em RDF para padrões de OAs não é uma tarefa trivial, e a literatura carece de uma solução para essa questão. Portanto, investigar-se-ão possíveis mapeamentos entre metadados em RDF e metadados no padrão IEEE-LOM. Uma vez que esses mapeamentos tenham sido feitos para o padrão IEEE-LOM, outros padrões baseados no IEEE-LOM, como o OBAA e o SCORM, poderão se beneficiar dessa solução, promovendo a flexibilidade desta abordagem no uso de padrões de construção de OAs.

\section{Considerações Finais}

Um grande desafio da Educação é adaptar o processo de ensino-aprendizagem aos estilos cognitivo e de aprendizagem de cada estudante. No contexto da sala de aula, as estratégias de ensino são voltadas para a turma, pois parece impraticável que o professor atenda as especificidades de aprendizagem de cada aluno. Nesse aspecto, a Informática pode contribuir muito na Educação.

Neste artigo, apresenta-se uma pesquisa em andamento. Propõe-se uma nova abordagem, baseada nas tecnologias da Web Semântica, para a recomendação inteligente de conteúdo web na criação de materiais e objetos de aprendizagem. Além disso, mostra-se como um curso online pode ser criado usando OAs recomendados por buscas que usam ontologias. Mesmo que haja necessidade de criar novos objetos para esse curso, o usuário pode se beneficiar de uma série de conteúdos da Web Semântica, usando algoritmos semânticos que retornam os recursos web mais apropriados para compor esses novos OAs.

Os resultados preliminares das técnicas de busca estão sendo comparados para serem avaliados e discutidos, afim de implementar os algoritmos definitivos para a otimização da recomendação de OAs e da busca semântica por recursos web anotados em RDF. Então, esta abordagem poderá ser combinada com os trabalhos no estado da arte que recomendam tipos diferentes de OAs, definidos por Graf, Kinshuk e Ives (2010), para adaptar o processo de ensino aos estilos de aprendizagem e cognitivo de cada aprendiz.

Portanto, a recomendação e criação otimizadas de OAs modeladas por esta abordagem permitem que o usuário crie um curso online dando como entrada apenas o programa de curso. O usuário também pode entrar com informações opcionais usadas na filtragem de OAs ou criar seus próprios OAs, os quais podem ser utilizados por outros profissionais da Educação. Assim, esta abordagem mostra-se uma solução promissora para a efetivação do uso de tecnologias na Educação. 


\section{Agradecimentos}

Os autores agradecem à Pró-Reitoria de Pesquisa e Pós-Graduação por todo o suporte prestado no desenvolvimento deste trabalho.

\section{Referências}

ADVANCED DISTRIBUTED LEARNING INITIATIVE. Sharable Content Object Reference Model (SCORM ${ }^{\mathrm{TM}}$ ). Advanced Distributed Learning, 2001.

ARAÚJO, R. D. et al. Autoria automática de objetos de aprendizagem a partir de captura multimídia e associação a estilos de aprendizagem. In: Anais do Simpósio Brasileiro de Informática na Educação, Anais...2014.

BERNERS-LEE, T. et al. The semantic web. Scientific american, v. 284, n. 5, p. 2837, 2001.

CASTRO, C. V.; SATLER, M. F. Modelo ontológico para la secuenciación de objetos de aprendizaje. Revista Iberoamericana de Tecnologías del/da Aprendizaje/ Aprendizagem, p. 63, 2010.

DI MARTINO, B. Semantic retrieval of learning objects with schema matching. Journal of e-Learning and Knowledge Society, v. 5, n. 3, 2009a.

DI MARTINO, B. Semantic web services discovery based on structural ontology matching. International Journal of Web and Grid Services, v. 5, n. 1, p. 46-65, $2009 b$.

DING, L. et al. Swoogle: a search and metadata engine for the semantic web. In: Proceedings of the thirteenth ACM international conference on Information and knowledge management, Anais...ACM, 2004.

DUVAL, E. 1484.12. 1: IEEE Standard for Learning Object Metadata. IEEE LTSC, 2002.

FININ, T. el al. Swoogle: Searching for knowledge on the Semantic Web. In: Proceedings of the National Conference on Artificial Intelligence, Anais...Menlo Park, CA; Cambridge, MA; London; AAAI Press; MIT Press; 1999, 2005.

FRANZ, T. et al. Triplerank: ranking semantic web data by tensor decomposition. Berlin: Springer, 2009.

GAETA, M. et al. An Approach To Personalized e-Learning. Journal of Education, Informatics \& Cybernetics, v. 11, n. 1, 2013.

GRAF, S.; KINSHUK; IVES, C. A Flexible Mechanism for Providing Adaptivity Based on Learning Styles in Learning Management Systems. In: 2010 IEEE 10th International Conference on Advanced Learning Technologies (ICALT), Anais... In: 2010 IEEE 10TH INTERNATIONAL CONFERENCE ON ADVANCED LEARNING TECHNOLOGIES (ICALT). jul. 2010.

JÁCOME JÚNIOR, L.; MENDES NETO, F. M.; DA SILVA, L. C. N. Uma Abordagem Baseada em Algoritmo Genético para Recomendação de Objetos de Aprendizagem Sensível ao Contexto do Estudante. In: Anais do Simpósio Brasileiro de Informática na Educação, Anais...2012. 
MENOLLI, A.; MALUCELLI, A.; REINEHR, S. Criação Semi-Automática de Objetos de Aprendizagem a partir de Conteúdos da Wiki. In: Anais do Simpósio Brasileiro de Informática na Educação, Anais...2011.

SILVA, D. H.; DORÇA, F. A. Uma Abordagem Automática para Personalização do Processo de Ensino Baseada em Estilos de Aprendizagem em Sistemas Adaptativos e Inteligentes para Educação a Distância. Revista Brasileira de Informática na Educação, v. 22, n. 2, p. 1-15, 2014.

VICCARI, R. et al. The OBAA proposal for learning objects supported by agents. In: Procs. Of MASEIE Workshop-AAMAS, Anais...2010.

WANG, R. et al. Re-ranking search results using semantic similarity. In: Fuzzy Systems and Knowledge Discovery (FSKD), 2011 Eighth International Conference on, Anais...IEEE, 2011.

WEI, W.; BARNAGHI, P.; BARGIELA, A. Rational research model for ranking semantic entities. Information Sciences, v. 181, n. 13, p. 2823-2840, 2011. 